\title{
The Association of Each Disability Based on the Three Sub-Categories of the Roland-Morris Disability Questionnaire during Hospitalization with Itself at 1 Year Postoperatively in Patients with Degenerative Lumbar Spinal Stenosis
}

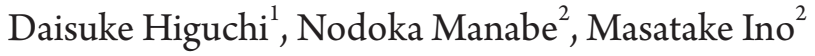 \\ ${ }^{1}$ Department of Rehabilitation, Harunaso Hospital, Takasaki, Japan \\ ${ }^{2}$ Gunma Spine Center, Kamitoyooka, Takasaki, Japan
}

\begin{abstract}
Study Design: A prospective study in a hospital.
Purpose: To investigate whether each disability based on the three sub-categories of the Roland-Morris disability questionnaire (RD0) during hospitalization is associated with itself at 1 year postoperatively in patients with degenerative lumbar spinal stenosis (LSS). Overview of Literature: Although the total score of the RDQ represents whole pain-related disabilities or health-related quality of life, it is a shortcoming that multi-dimensional changes in disabilities are difficult to understand when only using the RDO.

Methods: Fifty-seven patients with LSS (men, 28; women, 29; 63.0土12.1 years) were included. Disabilities, pain intensity and depressive feelings were assessed at preoperation, discharge and 1 year postoperatively.

Results: The range of "mental and physical activities (MPA)," "functional movements on/around a bed (FM)" and "walking function (WF)" scores were 0 to 13 (median, 8), 0 to 6 (median, 6) and 0 to 4 (median, 3) at preoperation; 0 to 12 (median, 0), 0 to 6 (median, 0), and 0 to 4 (median, 0) at discharge; and 0 to 8 (median, 0), 0 to 5 (median, 0), and 0 to 4 (median, 0) at 1 year postoperatively, respectively. The following significant multiple regression equations were obtained: MPA at 1 year postoperatively=0.56 (MPA at discharge)- 0.10 (depression at discharge) +0.90 (adjusted $r^{2}=0.41$ ), FM at 1 year postoperatively=0.35 (MPA at discharge)-0.06 (depression at discharge) +0.40 (adjusted $r^{2}=0.45$ ) and WF at 1 year postoperatively=0.59 (WF at discharge) -0.08 (depression at discharge) +0.63 (adjusted $r^{2}=0.29$ ).

Conclusions: In our LSS population, each disability based on MPA and WF at discharge is associated with itself in the future. Therefore, disabilities excluding functional movements are longitudinally independent.
\end{abstract}

Keywords: Activities of daily living; Lumbar vertebrae; Spinal stenosis; Roland-Morris disability questionnaire

\section{Introduction}

Lumbar spinal stenosis (LSS) is a syndrome caused by the acquired or congenital stenotic state of the lumbar spinal canal, nerve root canal or intervertebral foramina. Arnoldi et al. [1] classified acquired LSS into six types:

Received Oct 23, 2012; Revised Feb 7, 2013; Accepted Feb 12, 2013

Corresponding author: Daisuke Higuchi

Department of Rehabilitation, Harunaso Hospital,

5989 Nakamuroda, Takasaki, Gunma 370-3347, Japan

Tel: +81-27-374-1135, Fax: +81-27-374-1139, E-mail: evolution_19801208@hotmail.com 
degenerative, combined congenital and degenerative, spondylolisthetic/spondylitic, iatrogenic, post-traumatic and miscellaneous. Physical symptoms include low back pain, leg pain or numbness and paralysis of the leg. Conservative treatment is first selected; however, surgical treatment is applied when LSS symptoms progress.

The Roland-Morris disability questionnaire (RDQ) was developed to measure the degree of disability due to low back pain [2]. For the queries, Patrick et al. [3] then exchanged "because of my back" for "my back or leg pain" in order to include not only low back pain but neuropathic leg pain as well. Nakamura et al. [4] translated the modified RDQ presented by Patrick et al. [3] into Japanese. Ever since the RDQ has been published, many researchers have conducted studies to improve its usefulness [5-7].

Although it is widely accepted that the total score of the RDQ represents whole pain-related disabilities [7-10] or health-related quality of life $[3,11,12]$, it is a shortcoming that multi-dimensional changes in disabilities are difficult to understand by only using the RDQ. In addition, the Japanese Orthopaedic Association (JOA) and the Japanese Society for Spine Surgery and Related Research (JSSR) modified the JOA score for low back pain and named it the JOA Back Pain Evaluation Questionnaire (JOABPEQ) [13]. Unlike the JOA score, five sub-category scores of the JOABPEQ are never summed up because the JOA and the JSSR consider that the five sub-categories are independent, and therefore, adding up these scores is not meaningful. We attempted to categorize 24 items of the RDQ using a cluster analysis in preoperative patients with LSS and lumbar disc herniation. We then proposed three sub-categories, "mental and physical activities" (MPA), "functional movements on/around a bed" (FM) and "walking function" (WF) [14]. MPA consists of questions concerning the degree of activity (e.g., resting on a bed), activities of daily living (ADL) (e.g., changing clothes) and feelings and physiological desires (e.g., dependence on others and appetite); MPA display difficulties in performing social roles away from a bed. FM consists of movements not involving locomotion, such as postural changes and standing. Nagi [15] defined "disabilities" as a limitation in the performance of socially defined roles and tasks within a sociocultural and physical environment, and "functional limitations" as a limitation in performance at the level of the whole organism or person. Based on the concept of Nagi [15], MPA is similar to disabilities, and FM and WF are generally covered by functional limitations. For example, although changing clothes while sitting on a bed and simply sitting quietly on a bed are the same or similar in appearance, the former is considered a "disability" because tidying up oneself is an important action for successfully performing social roles. However, "sitting quietly on a bed" is considered a "functional limitation" because sitting is posture maintenance. Hence, it is required for longitudinal research to improve the validity for dividing the RDQ into three subcategories.

In the present study, we aim to investigate whether each disability based on the above mentioned three subcategories of the RDQ at discharge, when immediate recovery due to surgery is achieved, are associated with the same disability at 1 year postoperatively in patients with degenerative LSS. We also wish to confirm that the three disabilities are independent regardless of the time course.

\section{Materials and Methods}

\section{Study design}

The present study was prospective in nature and was conducted from July 2010 to June 2012 at Harunaso Hospital in Japan after gaining approval from the hospital ethics committee.

\section{Participants}

Patients included those who were diagnosed with LSS, which was considered a degenerative type by Arnoldi et al. [1], based on clinical and radiological findings and were admitted for surgery. Patients who had obvious motor and sensory dysfunction based on comorbidity or anamnesis before surgery, who had onset of other diseases after discharge, or who could not fully understand the nature of our investigation were excluded. Written informed consent to participate in the present study was obtained from all participants after sufficient explanation of the study protocol.

Surgical indications included inadequate response to conservative treatment, rapid neurological deterioration and marked limitation of ADL. As a general rule, in patients who had low back pain derived from the facet(s) or whose sagittal and/or coronal alignment of the spine was poor, decompression with fixation using instrumentation was performed. Further, in cases whose alignment was good, only decompression was applied. 


\section{Surgical procedure and postoperative treatment}

1) Decompression with instrumentation

Laminectomy and bilateral hemifacetectomy, depending on the patient's pathological state at the stenotic level, were performed through a midline posterior approach. Nearly complete discectomy was performed while a traversing nerve root was protected by sliding a retractor. Two square-type or one boomerang-type interbody spacers (PROSPACE Titanium or TSPACE Titanium, Aesculap AG, Tuttlingen, Germany) were then inserted, and bone grafting using local bones was performed. Finally, instrumentation using pedicle screws and connecting rods (EXPEDIUM 5.5 Spine System, DePuy Spine Inc., Raynham, MA, USA) was performed. Compression was applied throughout the instrumentation in order to restore lordosis.

\section{2) Only decompression}

Central decompression was performed by medial laminotomy or laminectomy depending on the patient's situation through a midline posterior approach. The facet(s) was preserved; however, foraminotomy was added, if necessary.

\section{3) Postoperative treatment}

Patients were permitted to sit and walk with a canvas corset after 2 or 3 days postoperatively and underwent postoperative physical therapy. When they could safely perform fundamental ADL, for example, walking and self-care, they were allowed to be discharged. After discharge, they were requested to avoid activities with excessive trunk motion in daily living.

\section{Assessment}

1) Disability

The RDQ translated into Japanese by Nakamura et al. [4] was used. Question nos. 1, 4, 6, 8, 9, 13, 15, 16, 18-22, and 24 were considered as "MPA"; nos. 2, 7, 10-12, and 14 were considered as "FM"; and nos. 3, 5, 17, and 23 were considered as "WF" [14].

\section{2) Pain intensity}

The intensity of low back pain and leg pain were measured by the 11-point numerical rating scale; 0 represented no pain and 10 was unbearable pain.

\section{3) Depressive feelings}

The degree of depressive feelings was measured by a part of the Hospital Anxiety and Depression Scale (HADS) [16]. Scores of depressive feeling item range from 0 to 21 points; higher scores represent severer depressive feelings.

The RDQ was assessed at preoperation, discharge and follow-up (FU) at 1 year postoperatively; all other items were assessed at preoperation and discharge.

\section{Analytical procedure}

First, the fundamental statistics of all assessed items were calculated; then, the assessed items were compared among preoperation, discharge and FU at 1 year postoperatively with the Steel-Dwass test or Wilcoxon signed rank test. Second, a stepwise multiple regression analysis was performed. MPA, FM and WF scores at FU were used as dependent variables and scores of the other items at discharge were used as independent variables. All statistics were performed using the R ver. 2.8 (http://www. r-project.org/). Values of $p<0.05$ were considered statistically significant.

\section{Results}

A total of 57 LSS patients (men, 28; women, 29; 63.0 \pm 12.1 [standard deviation] years) participated in the present study. Thirty-six patients (63.2\%) had LSS with degenerative spondylolisthesis, seven patients (12.3\%) had degenerative local kyphosis and/or scoliosis and three patients (5.3\%) had LSS with facet syndrome (some patients had overlapping conditions). Forty-four patients (77.2\%) underwent decompression with instrumentation and 13 patients $(22.8 \%)$ underwent only decompression. The mean number of days among assessments was $31.3 \pm 21.5$ from preoperation to discharge.

The fundamental statistics of all assessed items are summarized in Table 1. All assessed item, including MPA, FM, and WF scores of the RDQ at discharge, were significantly lower than those at preoperation $(p<0.01)$. MPA, FM, and WF scores were not significantly different between discharge and at 1 year postoperatively.

A stepwise multiple regression analysis indicated that the FU-MPA score was significantly correlated with the discharge (Dis)-MPA score and Dis-depression score. The FU-FM score was correlated with the Dis-MPA score and Dis-depression score. The FU-WF score was correlated 
Table 1. Fundamental statistics of all assessed items $(n=57)$

\begin{tabular}{|c|c|c|c|}
\hline Items & Preoperation & Discharge & $\begin{array}{l}\text { Follow-up } \\
\text { (1 year postoperatively) }\end{array}$ \\
\hline \multicolumn{4}{|l|}{ Roland-Morris disability questionnaire } \\
\hline Total score (0-24 points) & $8(4.0)[0-22]$ & $0(1.5)[0-22]^{a)}$ & $0(1.0)[0-17]^{b)}$ \\
\hline Mental and physical activities score (0-14 points) & $3(2.0)[0-13]$ & $0(0.5)[0-12]^{a)}$ & $0(0.5)[0-8]^{b)}$ \\
\hline Functional movements on/around a bed score ( $0-6$ points) & $2(1.5)[0-6]$ & $0(0.5)[0-6]^{a)}$ & $0(0.0)[0-5]^{b)}$ \\
\hline Walking functions score (0-4 points) & $3(1.0)[0-4]$ & $0(0.5)[0-4]^{a)}$ & $0(0.0)[0-4]^{b)}$ \\
\hline Low back pain (0-10 points) & $5(2.5)[0-10]$ & $0(1.0)[0-5]^{c)}$ & - \\
\hline Leg pain (0-10 points) & $5(2.5)[0-10]$ & $0(0.5)[0-8]^{c)}$ & - \\
\hline $\begin{array}{l}\text { Depression domain score of hospital anxiety and depression scale } \\
\text { (0-21 points) }\end{array}$ & $8(3.5)[0-20]$ & $6(2.0)[0-20]^{c)}$ & - \\
\hline
\end{tabular}

Scores mean median (quartile deviation) [range].

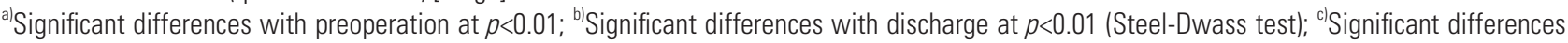
with at preoperation at $p<0.01$ (Wilcoxon signed rank test).

Table 2. Coefficients of multiple regression equations for sub-category scores of the Roland-Morris disability questionnaire at 1 year postoperatively $(n=57)$

\begin{tabular}{|c|c|c|c|c|c|c|}
\hline Independent variables & Dependent variables & PRC & $t$-value & SPRC & F-value & Adjusted $r^{2}$ \\
\hline \multirow[t]{4}{*}{ FU-MPA score } & & & & & $20.5^{\text {a) }}$ & 0.41 \\
\hline & Dis-MPA score & 0.56 & $6.40^{\text {a) }}$ & 0.68 & & \\
\hline & Dis-depression score & -0.10 & $-2.02^{b)}$ & -0.22 & & \\
\hline & & Constant: 0.90 & $2.62^{b)}$ & & & \\
\hline \multirow[t]{4}{*}{ FU-FM score } & & & & & $23.99^{\mathrm{al}}$ & 0.45 \\
\hline & Dis-MPA score & 0.35 & $6.92^{\text {a) }}$ & 0.71 & & \\
\hline & Dis-depression score & -0.06 & $-2.11^{b)}$ & -0.22 & & \\
\hline & & Constant: 0.40 & $2.01^{b)}$ & & & \\
\hline \multirow[t]{4}{*}{ FU-WF score } & & & & & $12.13^{\mathrm{a})}$ & 0.29 \\
\hline & Dis-WF score & 0.59 & $4.91^{\text {a) }}$ & 0.61 & & \\
\hline & Dis-depression score & -0.08 & $-2.32^{b)}$ & -0.29 & & \\
\hline & & Constant: 0.63 & $2.68^{\mathrm{bl}}$ & & & \\
\hline
\end{tabular}

PRC, partial regression coefficient; SPRC, standardized partial regression coefficient; FU, follow-up; MPA, mental and physical activity; Dis, discharge; FM, functional movement; WF, walking function.

a) $p<0.01 ;{ }^{\text {b) }} p<0.05$.

with the Dis-WF score and Dis-depression score (Table 2). There were no independent variables of which variation inflation factors were $>10$.

\section{Discussion}

\section{Characteristics of participants in the present study}

In the present study, the median preoperative total score of the RDQ and low back and leg pain scores were 8 
(quartile deviation $[\mathrm{QD}], 4.0), 5(\mathrm{QD}, 2.5)$, and $5(\mathrm{QD}$, $2.5)$, respectively. The degree of disability would be lower although the intensities of low back and leg pain would be equivalent or slighter in our cohort compared with previous studies [17-19].

In our study, all assessed items significantly improved from preoperation to discharge. We considered that disabilities and psychological problems improved according to a decrease in low back and leg pain due to LSS in the early postoperative period. Disabilities were not obviously improved from discharge to 1 year postoperatively.

\section{Longitudinal independence of the three sub-catego- ries of the RDQ}

F-values and t-values of all multiple regression equations were statistically significant. Adjusted $r^{2}$ were not high, which suggested a complicated process where LSS patients who were liberated from severe pain by surgery readapted to daily living based on the respective values or attitudes after discharge. Therefore, it was unsurprising that disabilities at 1 year postoperatively could not be mostly explained by only two variables, MPA or WF and the depression score at discharge. The present study aimed to confirm whether the three sub-categories of the RDQ were longitudinally independent rather than to obtain accurate prediction formulae for the sub-categories at 1 year postoperatively.

We presented that each disability based on MPA and WF of the RDQ as well as depressive feelings at discharge were independently associated with itself at 1 year postoperatively. This result supported the notion that MPA and WF of the RDQ would be longitudinally independent. However, the Dis-FM score was not associated with the FU-FM score against expectations, which could have been caused by differences in life style at the hospital and at home. Aged Japanese often prefer a "futon" (quilted Japanese-style mattress laid out on the floor); yet, operated LSS patients were requested to use a bed, not a "futon." Therefore, these patients had to change their style to a Western life style in order to avoid surplus stress to the operated lumbar vertebrae during their hospital stay. Consequently, functional movements performed by LSS patients after discharge would tend to disagree with those performed while being hospitalized. The finding that FM at 1 year postoperatively was not significantly correlated with FM at discharge did not indicate that the sub-cate- gory FM was not longitudinally independent due to differences in the living environments of LSS patients at the hospital and at home. Therefore, a further comparison of FM for the time points, excluding the effect of differences in life style, are required to guarantee the validity of categorizing the RDQ into three sub-categories.

Moreover, the Dis-depression domain score of the HADS affected all three sub-categories at the FU. Depressive feelings at discharge did not result in poorer, but better activities and functions at 1 year postoperatively. Sinikallio et al. [20] advocated that prior to an operation, depressive LSS patients were likely to have high subjective disability of everyday living and low life satisfaction. Considering these findings, we consider that LSS patients who had severe depressive feelings at discharge would feel more comfortable performing movements in daily living without low back and/or leg pain compared to nondepressive patients because depressive feelings improved after discharge.

From the view point of clinical application, individually assessing the three disabilities, MPA, FM, and WF, is useful for providing effective and appropriate rehabilitation as conservative or postoperative treatment for LSS patients. For example, patients who have decreased MPA may obtain more benefit from this rehabilitation program, including a behavioral psychological standpoint as cognitive-behavioral therapy to improve patients' activity level in the society. Those who have decreased FM or WF may benefit from this rehabilitation program, including advising how to change and maintain posture on a bed or how to walk without exacerbating the low back and/ or leg pain. Because we found that each disability based on MPA and WF at discharge was positively associated with itself at 1 year postoperatively, the physical therapy for these disabilities, as postoperative rehabilitation at the hospital, would directly and favorably affect the surgical outcome of LSS patients after discharge.

\section{Study limitations and future prospects}

This study focused on a small sample recruited from a single center in Japan. Thus, we restricted the number of variables that were used as dependent variables in the multiple regression analysis. The results from the present study should be interpreted with caution. In the future, larger sample sizes and multicenter studies should be required in order to obtain secure prediction formulae 
for the three sub-categories of the RDQ at 1 year postoperatively using many dependent variables. A comparison between races or countries should also be performed because there are large differences in life style and belief based on religion, as indicated by Jones et al. [21] who demonstrated that there are racial differences in the coping strategies for chronic arthralgia.

\section{Conclusions}

We followed up pre- and postoperative scores of the three sub-categories, "MPA," "FM," and "WF" of the RDQ in order to investigate whether each disability based on these sub-categories was longitudinally independent in patients with degenerative LSS. We obtained the following significant multiple regression equations: We think the following descriptions are better: MPA at 1 year postoperatively $=0.56$ (MPA at discharge)- 0.10 (depression at discharge) +0.90 (adjusted $r^{2}=0.41$ ), FM at 1 year postoperatively $=0.35$ (MPA at discharge) -0.06 (depression at discharge) +0.40 (adjusted $r^{2}=0.45$ ) and WF at 1 year postoperatively $=0.59$ (WF at discharge) -0.08 (depression at discharge) +0.63 (adjusted $r^{2}=0.29$ ). Each disability based on MPA and WF of the RDQ at discharge, when immediate postoperative recovery is acquired, is associated with itself at 1 year postoperatively. Therefore, disabilities, excluding functional movements (e.g., rolling and standing up), would be longitudinally independent, regardless of the time course.

\section{Conflict of Interest}

No potential conflict of interest relevant to this article was reported.

\section{References}

1. Arnoldi CC, Brodsky AE, Cauchoix J, et al. Lumbar spinal stenosis and nerve root entrapment syndromes. Definition and classification. Clin Orthop Relat Res 1976;(115):4-5.

2. Roland M, Morris R. A study of the natural history of back pain. Part I: development of a reliable and sensitive measure of disability in low-back pain. Spine (Phila Pa 1976) 1983;8:141-4.

3. Patrick DL, Deyo RA, Atlas SJ, Singer DE, Chapin A, Keller RB. Assessing health-related quality of life in patients with sciatica. Spine (Phila Pa 1976) 1995;20:1899-908.

4. Nakamura M, Miyamoto K, Shimizu K. Validation of the Japanese version of the Roland-Morris Disability Questionnaire for Japanese patients with lumbar spinal diseases. Spine (Phila Pa 1976) 2003;28:2414-8.

5. Stratford PW, Binkley JM. Measurement properties of the RM-18. A modified version of the Roland-Morris Disability Scale. Spine (Phila Pa 1976) 1997;22:241621.

6. Ostelo RW, de Vet HC, Knol DL, van den Brandt PA. 24-item Roland-Morris Disability Questionnaire was preferred out of six functional status questionnaires for post-lumbar disc surgery. J Clin Epidemiol 2004;57:268-76.

7. Turner JA, Fulton-Kehoe D, Franklin G, Wickizer TM, Wu R. Comparison of the Roland-Morris Disability Questionnaire and generic health status measures: a population-based study of workers' compensation back injury claimants. Spine (Phila Pa 1976) 2003;28:1061-7.

8. Magnussen L, Strand LI, Lygren H. Reliability and validity of the back performance scale: observing activity limitation in patients with back pain. Spine (Phila Pa 1976) 2004;29:903-7.

9. Licciardone JC, Kearns CM. Somatic dysfunction and its association with chronic low back pain, backspecific functioning, and general health: results from the OSTEOPATHIC Trial. J Am Osteopath Assoc 2012;112:420-8.

10. Pryce R, Johnson M, Goytan M, Passmore S, Berrington N, Kriellaars D. Relationship between ambulatory performance and self-rated disability in patients with lumbar spinal stenosis. Spine (Phila $\mathrm{Pa}$ 1976) 2012;37:1316-23.

11. Igarashi A, Kikuchi S, Konno S. Correlation between inflammatory cytokines released from the lumbar facet joint tissue and symptoms in degenerative lumbar spinal disorders. J Orthop Sci 2007;12:154-60.

12. Opara J, Szary S, Kucharz E. Polish cultural adaptation of the Roland-Morris Questionnaire for evaluation of quality of life in patients with low back pain. Spine (Phila Pa 1976) 2006;31:2744-6.

13. The Japanese Orthopaedic Association, the Japanese Society for Spine Surgery and Related Research. Manual of JOABPEQ and JOACMEQ. Tokyo: Nankodo; 2012. 
14. Higuchi D. Categorization of the 24 items of the Roland-Morris disability questionnaire and its clinical usefulness. Rinsho Seikei Geka 2012;47:551-7.

15. Nagi SZ. Disability concepts revisited: implication for prevention. In: Pope AM, Tarlov AR, editors. Disability in America: toward a national agenda for prevention. Washington, DC: National Academies Press; 1991. p.309-27.

16. Zigmond AS, Snaith RP. The hospital anxiety and depression scale. Acta Psychiatr Scand 1983;67:361-70.

17. Toyone T, Tanaka T, Kato D, Kaneyama R, Otsuka M. Patients' expectations and satisfaction in lumbar spine surgery. Spine (Phila Pa 1976) 2005;30:2689-94.

18. Nakamura M, Miyamoto K, Shimizu K. Difference in evaluation of patients with low back pain using the Japanese Orthopaedic Association Score for Back
Pain and the Japanese Version of the Roland-Morris Disability Questionnaire. J Orthop Sci 2009;14:36773.

19. Ohtori S, Ito T, Yamashita M, et al. Evaluation of low back pain using the Japanese Orthopaedic Association Back Pain Evaluation Questionnaire for lumbar spinal disease in a multicenter study: differences in scores based on age, sex, and type of disease. J Orthop Sci 2010;15:86-91.

20. Sinikallio S, Aalto T, Airaksinen O, et al. Depression and associated factors in patients with lumbar spinal stenosis. Disabil Rehabil 2006;28:415-22.

21. Jones AC, Kwoh CK, Groeneveld PW, Mor M, Geng $M$, Ibrahim SA. Investigating racial differences in coping with chronic osteoarthritis pain. J Cross Cult Gerontol 2008;23:339-47. 